


\title{
Two-electron relativistic corrections to the potential energy surface and vibration-rotation levels of water
}

\author{
Harry M. Quiney, \\ School of Chemistry, University of Melbourne, \\ Victoria 3010, Australia \\ Paolo Barletta \\ Department of Physics and Astronomy, \\ University College London, \\ Gower St, London WC1E 6BT, U.K. \\ György Tarczay, Attila G. Császár, \\ Department of Theoretical Chemistry, Eötvös University, \\ H-1518 Budapest 112, P.O. Box 32, Hungary \\ Oleg L. Polyansky, ${ }^{a}$ and Jonathan Tennyson \\ Department of Physics and Astronomy, \\ University College London, \\ Gower St, London WC1E 6BT, U.K.
}

${ }^{a}$ Permanent address: Institute of Applied Physics, Russian Academy of Sciences, Uljanov Street 46, Nizhnii Novgorod, Russia 603024.

April 17, 2005 


\begin{abstract}
Two-electron relativistic corrections to the ground-state electronic energy of water are determined at over 300 geometries. The corrections include the two-electron Darwin term of the Coulomb-Pauli Hamiltonian, and the Gaunt and Breit corrections, calculated perturbationally using four-component variational Dirac-Hartree-Fock wavefunctions. Fitted relativistic correction surfaces are constructed and used with an accurate $a b$ initio nonrelativistic Born-Oppenheimer potential to calculate vibrational and rotational levels for $\mathrm{H}_{2}{ }^{16} \mathrm{O}$. The calculations suggest that these two-electron relativistic corrections, which have so far been neglected in rovibrational calculations on light molecules, have a substantial influence on the levels of water. The effects considered have markedly different characteristics for the stretching and bending levels.
\end{abstract}




\section{Introduction}

Many decades of work have been performed measuring, analyzing, and modelling the rovibrational spectrum of water (see, for example, $[1,2,3,4]$ and references therein). Despite this considerable effort, much further work remains to be done. For example, Polyansky et al. [3, 5] recently assigned 1687 features in the spectrum of sunspots recorded in the $10-20 \mu \mathrm{m}$ region to transitions in water. These transitions represent only about $15 \%$ of the clearly resolved features observed in sunspots in this spectral region, and it is likely that nearly all of the unassigned features are also due to water. Further significant progress in assigning these features requires corresponding theoretical developments. In particular, it is now essential that the techniques of ab initio computational methods be extended to consider the many small physical effects which are usually neglected, but which may influence the measured transition frequencies of water.

The major factor determining the accuracy of a variationally calculated vibrationrotation spectrum is the potential energy surface (PES) employed. State-of-the-art $a b$ initio electronic structure calculations are now capable $[2,6,7]$ of predicting vibrational band origins (VBOs) and other spectroscopic properties of water with an accuracy of $\sim 0.1 \%$. To achieve this accuracy several physically significant factors, tacitly neglected in most works in computational molecular spectroscopy, must be considered: core-valence electron correlation, relativistic corrections, and coupling between electronic and nuclear motion, part of which is considered in the so-called Born-Oppenheimer diagonal correction (BODC) [8]. The validity of the Born-Oppenheimer approximation when calculating vibration-rotation spectra of water has been explored $[2,9,10]$. Relativistic effects $[11,12,13]$ have also been attracting considerable attention $[7,14,15]$. The effect of the so-called scalar relativistic correction, comprising the one-electron mass-velocity and Darwin corrections (MVD1), has been investigated in detail for the VBOs and rotational term values of water [14]. It has been assumed in the past that for a molecule such as water, the absolute relativistic energy correction may be significant but its variation with geometry is too small to be important. The most notable result of Ref. [14], however, was the demonstration of the sensitivity of the results to the inclusion of the dominant relativistic MVD1 correction to the PES of a light closed-shell molecular system. This should be contrasted with inclusion of the BODC, which only has a minor influence [9]. In general, addition of the MVD1 relativistic correction lowers the band origins of the stretching states but raises the band origins of the bending modes, as expected from the increased barrier to linearity of water found upon inclusion of relativistic effects $[16,17]$.

After the dominant one-electron mass-velocity and Darwin corrections, the next most 
significant spin-independent relativistic effect is the two-electron Darwin (D2) term; the sum of these terms, MVD2 = MVD1 + D2, defines the Coulomb-Pauli approximation. Spin-orbit interactions can be neglected for light closed-shell molecules and it is generally assumed that the Coulomb-Pauli Hamiltonian [11] yields good approximations to results obtained from variational four-component solutions of the many-electron relativistic Dirac-Coulomb equation $[11,18]$. For example, at the grid points of this study (vide infra) the maximum relative deviation between the MVD2 and Dirac-Hartree-Fock (DHF) energies is only $0.76 \mathrm{~cm}^{-1}$ [18].

In order to improve significantly on the description provided by the Dirac-Coulomb equation, or by the Coulomb-Pauli approximation to it, the instantaneous charge-charge interaction defining the Coulomb interaction must be supplemented by interactions between electronic currents, and Lorentz covariance must be restored to within some specified approximation by the inclusion of retardation effects. The covariant frequencydependent transverse Coulomb-gauge interaction is rather complicated in form, but is nevertheless used routinely in high-precision atomic structure studies. The low-frequency form of the Dirac-Coulomb-Breit Hamiltonian contains the leading-order quantum electrodynamic (QED) correction to the Coulomb interaction [13]. It offers a tractable approximation for detailed molecular studies, and incorporates all electronic terms correct to $O\left((Z \alpha)^{2}\right)$. From this may be derived the two-component Pauli approximation, introducing spin-dependent interactions in addition to the operators which define the scalar Coulomb-Pauli theory. The Dirac-Coulomb-Gaunt Hamiltonian includes only the magnetic interactions between pairs of electronic currents, neglecting certain $O\left((Z \alpha)^{2}\right)$ contributions $[13,19,20]$. Quiney et al. [20] have already probed the Gaunt and Breit energy corrections for water; their work and simple physical arguments suggest that the inclusion of the geometry dependence of the Breit correction in the ground-state PES of water should have a noticable effect on the calculated VBOs and rotational term values. It is this possibility which we principally address in this letter. At this point it should be noted that an even smaller correction due to the leading QED effect requiring renormalization of divergences, the one-electron Lamb-shift effect (self-energy and vacuum polarization [19]), has been investigated for the ground-state PES of water [15]. Estimates of the one-electron Lamb-shift in the PES yield corrections of up to $1 \mathrm{~cm}^{-1}$ in magnitude for the rovibrational states of water. Inclusion of the two-electron Lambshift effect in the PES has, on the other hand, negligible influence on the rovibrational states [15]. Similarly, a recent calculation has shown that spin-orbit interactions make a negligible contribution to the shape of the water ground state potential energy surface $[18]$. 


\section{Computational Techniques}

The energy correction due to the two-electron Darwin term, D2, has been computed with cc-pVQZ CCSD(T) $[21,22]$ wave functions, at the same level as the previous calculations of MVD1 correction energies [14].

Relativistic energy corrections due to the Gaunt and Breit interactions were obtained in first order of perturbation theory using the four-component Dirac-Hartree-Fock (DHF) wave function [13], the recommended exponent factors for the Gaussian nuclear charge distribution [23], and the following [O, $\mathrm{H}]$ basis sets: basis $\mathrm{A}=[11 s 6 p, 6 s]$ and basis $\mathrm{B}=[11 s 6 p 3 d, 6 s 3 p]$ for the large component. The restricted kinetic balance prescription [13] was used to generate the small-component basis functions from the large component set in a one-to-one mapping. The calculations have been repeated at over 300 structures comprising the data set $1.47 \leq$ distance $\leq 2.79 a_{0}$ and $41 \mathrm{deg} \leq$ angle $\leq 172$ deg. The computer codes DIRCCR12 [24], MOLFDIR [25, 26], and BERTHA $[13,27]$ have been employed for the electronic structure calculations involving the D2, Gaunt, and Breit terms, respectively. Values for each energy correction obtained with basis set B have been placed on our web site, see below.

The absolute values of the (Gaunt,Breit) energy correction on the PES of water is about $(7.8,7.6) \mathrm{m} E_{\mathrm{h}}$, while the maximum difference within the region covered by our grid is $(46,42) \mathrm{cm}^{-1}$. The D2 effect is smaller, being $3.3 \mathrm{~m} E_{\mathrm{h}}$ and $6.5 \mathrm{~cm}^{-1}$, respectively. Figure 1 shows how the two-electron Darwin, Gaunt, and the Breit corrections vary as a function of bond angle and the symmetric stretching coordinate.

In order to use the calculated relativistic corrections in nuclear motion calculations we have fitted them to an analytic functional form which is the same as the one used in Ref. [14]. The computer algebra package Mathematica [28, 29] was used for the fitting and for the automatic generation of the PES subroutines in FORTRAN. The 55 coefficients obtained from a least-squares fit to our data points can be downloaded from the web site ftp://ftp.tampa.phys.ucl.ac.uk/pub/vr/potentials/H2O.rel, and are incorporated within FORTRAN routines representing the PESs. The fit gives an accurate representation of the data and has a standard deviation of only $0.02 \mathrm{~cm}^{-1}$ or better.

Nuclear motion calculations were performed using the DVR3D program suite [30] and previously optimized basis sets [31]. Calculations were only performed for the $\mathrm{H}_{2}^{16} \mathrm{O}$ isotopomer of water. All calculations presented here used a hydrogen mass midway between the atomic and nuclear value, as recommended by Zobov et al. [9]. 


\section{Discussion}

Tables 1 and 2 summarize calculations for selected vibrational and rotational term values of water, respectively. These calculations were all performed with PESs being a sum of non-relativistic and relativistic correction surfaces, where the nonrelativistic surface is the $a b$ initio Born-Oppenheimer (BO) surface of Partridge and Schwenke [2] ${ }^{1}$ corrected with the mass-dependent BODC correction of Zobov et al. [9]. To maintain consistency, the non-relativistic results are the same as given in Ref. [14].

Relativistic corrections to the PES can either raise or lower the rovibrational bands. To understand the observed relativistic shifts in the bending band origins it is worth discussing relativistic effects on the barrier to linearity of water and on the one-dimensional bending functions. Recent studies $[7,14,15,18,20]$, as well as the present one, indicate that the one-electron kinetic relativistic effect (MVD1) raises the barrier by about 55 $\mathrm{cm}^{-1}$, while the D2 term raises the barrier by $2.5 \mathrm{~cm}^{-1}$. Both the MVD1 and the D2 bending curves show monotonic behavior. Beyond these scalar relativistic effects, the Gaunt correction raises the barrier by $6 \mathrm{~cm}^{-1}$, the correction to it in the Breit operator compensates this effect by $2 \mathrm{~cm}^{-1}$, and consequently the Breit correction raises the barrier by $4 \mathrm{~cm}^{-1}$. The approximation to the Lamb-shift correction [15] lowers the barrier by almost $4 \mathrm{~cm}^{-1}$. As seen below, these changes in the PES mostly translate directly into shifts of the computed bending band origins.

Our results clearly indicate that the two-electron relativistic corrections considered in this study have a significant influence on the calculated behavior of both the vibrational and rotational states of water. However, these results are fairly insensitive to the level of sophistication of the calculation. The mean deviation between the changes in the Gaunt VBOs determined with basis $\mathrm{A}$ and basis $\mathrm{B}$ is only $0.05 \mathrm{~cm}^{-1}$, or approximately $2 \%$, with the larger basis B corrections giving slightly larger values. The results for the Breit correction are similar, indicating that their effects are strongly localized near the nuclei.

The MVD1 relativistic corrections [14] for the pure bending levels grow faster than linearly, a good approximate formula for them is $1.221+0.024 n_{2}^{3}$, where $n_{2}$ is the bending quantum number. The linear formula $+1.4 n_{2}$ is a good approximation up to $n_{2}=3$. There is a rapid linear increase in the stretching corrections, which is well approximated by $-2.8\left(n_{1}+n_{3}\right)$, where $n_{1}$ and $n_{3}$ are stretching quantum numbers. Additivity of the stretch and bend corrections seems to hold to better than $95 \%$.

The average effect of inclusion of the relativistic two-electron correction terms in the

\footnotetext{
${ }^{1}$ Here we used the Partidge and Schwenke's best fit to their ab initio data as defined by the parameter $\left(c^{5 Z}, c^{\text {basis }}, c^{\text {core }}, c^{\text {fit }}\right)=(1,0,-1,0)$ in their potential.
} 
PES on the VBOs of water is not particularly large, considerably smaller than the effect arising from the inclusion of the MVD1 term, but appears to be significant.

The correction from D2 for the pure stretching VBOs is smaller than $0.2 \mathrm{~cm}^{-1}$ for the region covered here, and can be approximated well, up to $n_{1}+n_{3}=5$, by the relation $-0.01-0.04\left(n_{1}+n_{3}\right)$. The $\mathrm{D} 2$ correction increases for the bending modes with increasing excitation, it reaches the substantial value of $+0.8 \mathrm{~cm}^{-1}$ for $n_{2}=7$ and can be represented with the simple linear form $-0.07+0.12 n_{2}$. For stretch-bend combination levels the stretching and bending corrections seem to be additive to a good approximation; for example, the corrections for (100), (020), and (120) are $+0.05,-0.17$, and $-0.12 \mathrm{~cm}^{-1}$, respectively.

The Gaunt correction for the bending VBOs does not seem to follow a simple pattern. First it increases, peaks at about $n_{2}=3$ at $+0.21 \mathrm{~cm}^{-1}$, and decreases to $-0.15 \mathrm{~cm}^{-1}$ at $n_{2}=6$. The Gaunt correction is much larger for the stretching levels, grows linearly with excitation, and is well approximated as $-0.8\left(n_{1}+n_{3}\right) \mathrm{cm}^{-1}$. Similarly to the behavior of the D2 correction, there seems to be an additivity of stretching and bending Gaunt correction for the stretch-bend combination levels.

The Breit corrections are always smaller, except for the pure bending VBOs, than the Gaunt corrections, in most cases by some 20-30\%. The retardation correction, defined as Breit - Gaunt, is linear for the stretching modes, $0.15\left(n_{1}+n_{3}\right)$. The retardation correction for the bending modes varies, up to $n_{2}=3$, as $-0.02 n_{2}$, and it seems to decrease faster than linear after this.

Table 2 shows the $J=20$ rotational term values for the vibrational ground state calculated using the same models analysed above for the VBOs. The effect of the inclusion of two-electron relativistic corrections on the rotational term values is interestingly rather small as it would appear that the three contributions we consider here: D2, Gaunt, and Breit - Gaunt, approximately cancel each other out.

\section{Conclusions}

In this letter we have calculated $a b$ initio the contribution of various two-electron relativistic correction terms to the potential energy surface of water and their consequence on the vibration-rotation energy levels. Using this information it is possible to quantify the contributions of various terms which are neglected in a standard non-relativistic BornOppenheimer Schrödinger treatment of the electronic structure problem. For water the largest contribution to the vibrational band origins that have been assigned arises from the scalar one-electron relativistic correction, given by the one-electron mass-velocity 
plus Darwin (MVD1) terms, and it is $-19 \mathrm{~cm}^{-1}$, while the two-electron Darwin term (D2) contributes only $+0.8 \mathrm{~cm}^{-1}$, the Gaunt term contributes $-5 \mathrm{~cm}^{-1}$, and the Breit (= Gaunt + retardation) term contributes $-4 \mathrm{~cm}^{-1}$. These can be compared with Lamb shift effects which contribute a maximum of $+1.3 \mathrm{~cm}^{-1}$ [15], the adiabatic correction (or BODC) which contributes $+5 \mathrm{~cm}^{-1}$ and the non-adiabatic correction contributes -4 $\mathrm{cm}^{-1}$. In considering these numbers it should be remembered that lack of convergence of the best non-relativistic Born-Oppenheimer electronic structure calculations give an error of up to $30 \mathrm{~cm}^{-1}$ in the vibrational band origins.

Some important points should be noted about the above contributions. First, the maximum contribution does not distinguish between the behaviour of the bending and stretching modes, although for nearly all cases the magnitude and the sign of the contribution is mode dependent. For example, the error in the electronic structure calculation is predominantly in the bending mode [2]. Second, the corresponding contributions to the pure rotational energies are rather small so that the net effect is that two-electron relativistic effects contribute little. Finally, the differing signs of the various contributions may lead to a fortuitous cancellation of errors, and results whose agreement with the observations is superficial, and possibly misleading with respect to the accuracy of the individual contributions.

\section{Acknowledgements}

The work of PB, OLP, and JT was supported by the UK Engineering and Physical Science Research Council under grant GR/K47702, and the Russian Fund for Fundamental Studies. The work of GT and AGC has been supported by the Hungarian Ministry of Culture and Education (FKFP 0117/1997) and by the Scientific Research Foundation of Hungary (OTKA T024044 and T033074). Scientific exchanges between London and Budapest were supported by the Hungarian-British Joint Academic and Research Programme (project no. 076). This work was partially carried out on the Bentham supercomputer, at the HiPerSPACE Computing Centre, UCL.

\section{References}

[1] O. L. Polyansky, P. Jensen, J. Tennyson, J. Chem. Phys. 105 (1996) 6490.

[2] H. Partridge, D. W. Schwenke, J. Chem. Phys. 106 (1997) 4618. 
[3] O. L. Polyansky, N. F. Zobov, S. Viti, J. Tennyson, P. F. Bernath, L. Wallace, J. Molec. Spectrosc. 186 (1997) 422.

[4] A. G. Császár, I. M. Mills, Spectrochimica Acta 53A (1997) 1101.

[5] O. L. Polyansky, N. F. Zobov, S. Viti, J. Tennyson, P. F. Bernath, L. Wallace, Science 277 (1997) 346.

[6] A. G. Császár, W. D. Allen, Y. Yamaguchi, H. F. Schaefer III, in: P. Jensen, P. R. Bunker (Eds.), Computational Molecular Spectroscopy, Wiley, Chichester, 2000.

[7] A. G. Császár, G. Tarczay, M. L. Leininger, O. L. Polyansky, J. Tennyson, W. D. Allen, in: J. Demaison, K. Sarka (Eds.), Spectroscopy from Space, NATO ASI Series C, Kluwer, Dordrecht, 2001.

[8] N. C. Handy, Y. Yamaguchi, H. F. Schaefer III, J. Chem. Phys. 84 (1986) 4481.

[9] N. F. Zobov, O. L. Polyansky, C. R. Le Sueur, J. Tennyson, Chem. Phys. Lett. 260 (1996) 381.

[10] D. W. Schwenke, J. Phys. Chem. A, 105 (2001) 2352.

[11] K. Balasubramanian, Relativistic effects in Chemistry, Part A: Theory and techniques and Part B: Applications, Wiley, New York, 1997.

[12] P. Pyykkö, Chem. Rev. 88 (1988) 563 and the electronic bibliography http://www.csc.fi/lul/rtam.

[13] H. M. Quiney, H. Skaane, I. P. Grant, Adv. Quant. Chem. 32 (1999) 1.

[14] A. G. Császár, J. S. Kain, O. L. Polyansky, N. F. Zobov, J. Tennyson, Chem. Phys. Lett. 293 (1998) 317; 312 (1999) 613 (E).

[15] P. Pyykkö, K. Dyall, A. G. Császár, G. Tarczay, O. L. Polyansky, J. Tennyson, Phys. Rev. A 63 (2001) 024502.

[16] A. G. Császár, W. D. Allen, H. F. Schaefer III, J. Chem. Phys. 108 (1998) 9751.

[17] G. Tarczay, A. G. Császár, W. Klopper, V. Szalay, W. D. Allen, H. F. Schaefer III, J. Chem. Phys. 110 (1999) 11971.

[18] G. Tarczay, A. G. Császár, W. Klopper, H. M. Quiney, Mol. Phys. submitted, and references therein. 
[19] H. A. Bethe, E. E. Salpeter, Quantum Mechanics of One- and Two-Electron Atoms, Springer-Verlag, Berlin, 1957.

[20] H. M. Quiney, H. Skaane, I. P. Grant, Chem. Phys. Lett. 290 (1998) 473.

[21] T. H. Dunning, Jr., J. Chem. Phys. 90 (1989) 1007.

[22] K. Raghavachari, G. W. Trucks, J. A. Pople, M. Head-Gordon, Chem. Phys. Lett. 157 (1989) 479.

[23] Relativistic Effects in Heavy Element Chemistry (REHE, A program of the European Science Foundation) Newsletter No. 13, http://www.chemie.unierlangen.de/hess/html/esf/nl.html, 1995.

[24] DIRCCR12-95, an integral-direct explicitly correlated coupled-cluster program written by J. Noga and W. Klopper.

[25] MOLFDIR, P. J. C. Aerts, O. Visser, L. Visscher, H. Merenga, W. A. de Jong, W. C. Nieuwpoort, University of Groningen, The Netherlands.

[26] L. Visscher, O. Visser, P. J. C. Aerts, H. Merenga, W. C. Nieuwpoort, Comp. Phys. Commun. 81 (1994) 120.

[27] I. P. Grant, H. M. Quiney, Int. J. Quantum Chem. 80 (2000) 283.

[28] Mathematica, Version 2.2, Wolfram Research, Inc. (1993).

[29] S. Wolfram, Mathematica: A System for Doing Mathematics by Computers, 2nd ed., Addison-Wesley, Redwood City, CA, 1991.

[30] J. Tennyson, J. R. Henderson, N. G. Fulton, Comp. Phys. Comms. 86 (1995) 175.

[31] O. L. Polyansky, P. Jensen, J. Tennyson, J. Chem. Phys. 101 (1994) 7651.

[32] J. Tennyson, N. F. Zobov, R. Williams, O. L. Polyansky P. F. Bernath, J. Phys. Chem. Ref. Data, in press. 
Table 1

Vibrational band origins, in $\mathrm{cm}^{-1}$, for $\mathrm{H}_{2}^{16} \mathrm{O}$. Absolute values are given for the observed (Obs) VBOs and for the ab initio PES corresponding to the BornOppenheimer $(\mathrm{BO})+$ Born-Oppenheimer Diagonal Correction $\left(\Delta V_{\mathrm{ad}}\right)$ surface, and increments are given for the relativistic one-electron mass-velocity and Darwin (MVD1, $\left.+\Delta V_{\text {rel }}\right)$, the two-electron Darwin (+D2), the Gaunt (+Gaunt), and the retardation (+Retard., Breit - Gaunt) surface corrections. Increments are given as individual contributions.

\begin{tabular}{|c|c|c|c|c|c|c|}
\hline & $\mathrm{Obs}^{a}$ & $\mathrm{BO}+\Delta V_{\mathrm{ad}}$ & $+\Delta V_{\text {rel }}$ & $+\mathrm{D} 2$ & +Gaunt & +Retard. \\
\hline$(010)$ & 1594.75 & 1596.90 & 1.28 & 0.09 & -0.10 & -0.01 \\
\hline$(020)$ & 3151.63 & 3155.77 & 2.72 & 0.18 & -0.18 & -0.03 \\
\hline (100) & 3657.05 & 3660.48 & -2.80 & -0.05 & -0.78 & 0.15 \\
\hline (030) & 4666.80 & 4672.84 & 4.38 & 0.28 & -0.21 & -0.07 \\
\hline (110) & 5235.00 & 5240.67 & -1.56 & 0.04 & -0.89 & 0.15 \\
\hline (040) & 6134.03 & 6141.91 & 6.38 & 0.40 & -0.20 & -0.13 \\
\hline (120) & 6775.10 & 6782.80 & -0.20 & 0.13 & -0.96 & 0.12 \\
\hline (200) & 7201.54 & 7207.84 & -5.60 & -0.09 & -1.56 & 0.29 \\
\hline$(002)$ & 7445.07 & 7450.15 & -5.79 & -0.09 & -1.65 & 0.32 \\
\hline$(050)$ & 7542.39 & 7552.13 & 8.96 & 0.54 & -0.10 & -0.19 \\
\hline (130) & 8273.98 & 8283.66 & 1.38 & 0.23 & -1.01 & 0.09 \\
\hline (210) & 8761.59 & 8770.17 & -4.42 & -0.01 & -1.66 & 0.29 \\
\hline$(060)$ & 8870.50 & 8881.89 & 12.62 & 0.73 & 0.16 & -0.27 \\
\hline$(012)$ & 9000.14 & 9007.43 & -4.73 & -0.01 & -1.77 & 0.32 \\
\hline$(220)$ & 10284.37 & 10294.93 & -3.16 & 0.07 & -1.75 & 0.28 \\
\hline$(300)$ & 10599.69 & 10607.86 & -8.38 & -0.14 & -2.32 & 0.44 \\
\hline (102) & 10868.88 & 10877.70 & -8.50 & -0.14 & -2.37 & 0.45 \\
\hline$(310)$ & 12139.20 & 12149.75 & -7.29 & -0.06 & -2.44 & 0.44 \\
\hline (112) & 12407.64 & 12418.82 & -6.81 & 0.07 & -1.97 & 0.27 \\
\hline$(400)$ & 13828.28 & 13837.75 & -11.06 & -0.18 & -3.06 & 0.58 \\
\hline (122) & 13910.90 & 13924.10 & -6.22 & 0.02 & -2.57 & 0.44 \\
\hline$(202)$ & 14221.16 & 14233.05 & -11.19 & -0.18 & -3.10 & 0.58 \\
\hline$(004)$ & 14537.50 & 14547.06 & -11.59 & -0.19 & -3.25 & 0.62 \\
\hline$(330)$ & 15108.24 & 15122.47 & -5.03 & 0.10 & -2.62 & 0.40 \\
\hline$(410)$ & 15344.50 & 15356.30 & -9.92 & -0.10 & -3.17 & 0.58 \\
\hline$(212)$ & 15742.80 & 15757.01 & -10.12 & -0.11 & -3.20 & 0.59 \\
\hline$(302)$ & 17458.35 & 17471.62 & -13.88 & -0.23 & -3.84 & 0.73 \\
\hline$(510)$ & 18392.97 & 18405.23 & -12.11 & -0.12 & -3.83 & 0.71 \\
\hline$(034)$ & 18977.30 & 18993.66 & -9.27 & 0.00 & -3.63 & 0.61 \\
\hline$(520)$ & 19864.10 & 19877.85 & -11.82 & -0.07 & -4.01 & 0.72 \\
\hline (610) & 21221.57 & 21235.42 & -14.30 & -0.11 & -4.48 & 0.77 \\
\hline
\end{tabular}


Table 1, continued

\begin{tabular}{rrrrrrr}
\hline & $\mathrm{Obs}^{a}$ & $\mathrm{BO}+\Delta V_{\text {ad }}$ & $+\Delta V_{\text {rel }}$ & $+\mathrm{D} 2$ & +Gaunt & +Retard. \\
\hline$(001)$ & 3755.93 & 3758.30 & -2.90 & -0.05 & -0.84 & 0.16 \\
$(011)$ & 5331.27 & 5335.77 & -1.73 & 0.04 & -0.95 & 0.16 \\
$(021)$ & 6871.52 & 6878.15 & -0.45 & 0.12 & -1.04 & 0.14 \\
$(101)$ & 7249.80 & 7255.19 & -5.68 & -0.09 & -1.60 & 0.30 \\
$(031)$ & 8373.80 & 8382.57 & 1.03 & 0.22 & -1.09 & 0.11 \\
$(111)$ & 8807.00 & 8814.62 & -4.56 & -0.02 & -1.71 & 0.30 \\
$(041)$ & 9833.60 & 9844.25 & 2.80 & 0.32 & -1.10 & 0.06 \\
$(121)$ & 10328.70 & 10338.35 & -3.37 & 0.07 & -1.80 & 0.29 \\
$(201)$ & 10613.40 & 10621.12 & -8.42 & -0.14 & -2.34 & 0.44 \\
$(003)$ & 11032.40 & 11039.71 & -8.70 & -0.14 & -2.46 & 0.47 \\
$(131)$ & 11813.20 & 11824.90 & -2.01 & 0.15 & -1.87 & 0.26 \\
$(211)$ & 12151.30 & 12161.28 & -7.36 & -0.06 & -2.46 & 0.44 \\
$(301)$ & 13830.94 & 13840.36 & -11.06 & -0.18 & -3.07 & 0.58 \\
$(071)$ & 13835.37 & 13852.00 & 12.46 & 0.82 & -0.52 & -0.16 \\
$(023)$ & 14066.19 & 14078.00 & -6.75 & 0.00 & -2.70 & 0.46 \\
$(103)$ & 14318.81 & 14329.06 & -11.38 & -0.19 & -3.17 & 0.60 \\
$(231)$ & 15119.03 & 15132.98 & -5.10 & 0.09 & -2.64 & 0.41 \\
$(311)$ & 15347.96 & 15359.55 & -10.06 & -0.11 & -3.19 & 0.59 \\
$(033)$ & 15534.71 & 15548.74 & -5.60 & 0.08 & -2.78 & 0.44 \\
$(113)$ & 15832.76 & 15845.33 & -10.43 & -0.11 & -3.29 & 0.61 \\
$(321)$ & 16821.63 & 16834.24 & -9.93 & -0.08 & -3.39 & 0.60 \\
$(203)$ & 16898.84 & 16909.48 & -12.98 & -0.20 & -3.71 & 0.70 \\
$(123)$ & 17312.54 & 17326.99 & -9.48 & -0.04 & -3.40 & 0.60 \\
$(401)$ & 17495.53 & 17507.93 & -13.97 & -0.23 & -3.87 & 0.74 \\
$(331)$ & 18265.82 & 18280.83 & -8.66 & 0.01 & -3.46 & 0.58 \\
$(411)$ & 18393.30 & 18405.78 & -12.02 & -0.12 & -3.82 & 0.71 \\
$(213)$ & 18989.96 & 19004.62 & -13.03 & -0.16 & -3.99 & 0.75 \\
$(501)$ & 19781.10 & 19791.80 & -15.80 & -0.25 & -4.43 & 0.84 \\
$(511)$ & 21221.83 & 21235.74 & -14.32 & -0.15 & -4.48 & 0.83 \\
\hline
\end{tabular}

${ }^{a}$ Observed fundamentals are taken from ref [32]. All two-electron corrections are referenced to the Born-Oppenheimer $(\mathrm{BO})+$ Born-Oppenheimer Diagonal Correction $\left(\Delta V_{\mathrm{ad}}\right)+$ relativistic MVD1 $\left(\Delta V_{\text {rel }}\right)$ surface result. 
Table 2

Rotational term values $(J=20)$, in $\mathrm{cm}^{-1}$, for the vibrational ground state of $\mathrm{H}_{2}{ }^{16} \mathrm{O}$.

\begin{tabular}{lrrrrrr}
\hline & Obs $^{a}$ & BO+ $\Delta V_{\text {ad }}$ & $+\Delta V_{\text {rel }}$ & + D2 & +Gaunt & +Retard. \\
\hline $20_{020}$ & 4048.252 & 4048.396 & -1.264 & 0.884 & -1.060 & 0.175 \\
$20_{119}$ & 4412.316 & 4412.458 & -1.363 & 0.963 & -1.154 & 0.190 \\
$20_{218}$ & 4738.620 & 4738.806 & -1.356 & 1.021 & -1.226 & 0.201 \\
$20_{317}$ & 5031.798 & 5032.044 & -1.260 & 1.065 & -1.280 & 0.207 \\
$20_{416}$ & 5292.107 & 5292.440 & -1.014 & 1.089 & -1.313 & 0.208 \\
$20_{515}$ & 5513.235 & 5513.708 & -0.399 & 1.073 & -1.301 & 0.196 \\
$20_{614}$ & 5680.793 & 5681.431 & 0.575 & 1.015 & -1.243 & 0.171 \\
$20_{713}$ & 5812.071 & 5812.728 & 0.551 & 1.040 & -1.272 & 0.175 \\
$20_{812}$ & 5966.826 & 5967.311 & -0.838 & 1.189 & -1.440 & 0.223 \\
$20_{911}$ & 6170.841 & 6171.179 & -2.281 & 1.353 & -1.622 & 0.273 \\
$20_{1010}$ & 6407.447 & 6407.716 & -3.398 & 1.490 & -1.778 & 0.314 \\
$20_{119}$ & 6664.173 & 6664.418 & -4.417 & 1.623 & -1.927 & 0.352 \\
$20_{128}$ & 6935.419 & 6935.671 & -5.397 & 1.749 & -2.074 & 0.390 \\
$20_{137}$ & 7217.573 & 7217.850 & -6.377 & 1.880 & -2.221 & 0.427 \\
$20_{146}$ & 7507.580 & 7507.923 & -7.336 & 2.003 & -2.367 & 0.464 \\
$20_{155}$ & 7802.714 & 7803.154 & -8.311 & 2.133 & -2.511 & 0.501 \\
$20_{164}$ & 8100.284 & 8100.871 & -9.262 & 2.250 & -2.654 & 0.538 \\
$20_{173}$ & 8397.645 & 8398.419 & -10.241 & 2.376 & -2.795 & 0.574 \\
$20_{182}$ & 8691.921 & 8692.929 & -11.204 & 2.488 & -2.933 & 0.610 \\
$20_{191}$ & 8979.884 & 8981.191 & -12.204 & 2.607 & -3.069 & 0.646 \\
$20_{200}$ & 9257.451 & 9259.161 & -13.223 & 2.717 & -3.202 & 0.681 \\
\hline
\end{tabular}

${ }^{a}$ Observed rotational term values are taken from ref. [32]. For explanation of column headings see Table 1. 


\section{Figure captions}

\section{Figure 1}

Contour plot of two-electron relativistic correction surfaces as a function of the bond angle (in degrees) and the symmetric stretching (in $\AA$ ) coordinates. (a) Two-electron Darwin (D2) surface. The contour lines are separated by $1 \mathrm{~cm}^{-1}$ with a maximum at the top of the figure. (b) Gaunt interaction surface. The contour lines are separated by 2.5 $\mathrm{cm}^{-1}$, decreasing from left to right. (c) Breit - Gaunt interaction surface. The contour lines are separated by $1 \mathrm{~cm}^{-1}$, increasing from left to right. (d) Breit interaction surface. The contour lines are separated by $2.5 \mathrm{~cm}^{-1}$, decreasing from left to right. 